\title{
Atratividade de genótipos de feijão-caupi para oviposição de Bemisia tabaci biótipo B
}

\author{
Patrícia Leite Cruz(1), Edson Luiz Lopes Baldin(1), Maria de Jesus Passos de Castro(1), \\ Thiago Luis Martins Fanela(1) e Paulo Henrique Soares da Silva ${ }^{(2)}$
}

\begin{abstract}
(1)Universidade Estadual Paulista, Faculdade de Ciências Agronômicas, Campus de Botucatu, Rua José Barbosa de Barros, 1.780, Caixa Postal 237, CEP 18610-307 Botucatu, SP. E-mail: patricialeite@fca.unesp.br, elbaldin@fca.unesp.br, mjpcastro@fca.unesp.br, tImfanela@fca.com.br (2)Empresa Brasileira de Pesquisa Agropecuária, Centro de Pesquisa Agropecuária do Meio-Norte, Avenida Duque de Caxias, 5.650, Buenos Aires, Caixa Postal 01, CEP 64006-220 Teresina, PI. E-mail: phsilva@cpamn.embrapa.br
\end{abstract}

Resumo - O objetivo deste trabalho foi avaliar a atratividade de genótipos de feijão-caupi para oviposição de Bemisia tabaci biótipo $\mathrm{B}$ e identificar possíveis fontes de resistência à mosca-branca. Foram avaliados 51 genótipos, com uso de testes de chance de escolha. Os genótipos foram divididos aleatoriamente em dois grupos, tendo-se utilizado o genótipo Canapu como testemunha sucetível. Os 14 genótipos mais promissores (sete de cada grupo) foram selecionados para a realização de ensaios complementares (com ou sem chance de escolha). No teste com chance de escolha, os genótipos BRS-Urubuquara, TVU-36, TE93-244-23 F-1, BR 17-Gurgueia, BRS-Marataoã, MNC99-541 F-21 e TE97-304 G-4 foram menos atrativos à mosca-branca. Os genótipos TE93-244-23 F-1 e TVU-36 apresentaram resistência pelo mecanismo de não preferência para ovoposição. No teste sem chance de escolha, apenas o genótipo TVU-36 apresentou resistência por esse mecanismo.

Termos para indexação: Vigna unguiculata, antixenose, mosca-branca, resistência de plantas a insetos.

\section{Attractiveness of cowpea genotypes for Bemisia tabaci B-biotype oviposition}

\begin{abstract}
The objective of this work was to evaluate the attractiveness of cowpea genotypes for Bemisia tabaci B-biotype oviposition, and to identify possible sources of resistance. Fifty-one genotypes were evaluated using free-choice tests. The genotypes were randomly divided into two groups, using the genotype Canapu as a susceptible control. The fourteen most promising genotypes (seven from each group) were selected for complementary testing (with or without choice). In the free-choice test, BRS-Urubuquara, TVU-36, TE93-244-23 F-1, BR 17-Gurgueia, BRS-Marataoã, MNC99-541 F-21, and TE97-304 G-4 were less attractive to the whitefly. The genotypes TE93-244-23 F-1 and TVU-36 stud out and showed non-preference resistance for ovoposition. In the no-choice test, the genotype TVU-36 showed resistance by this same mechanism.
\end{abstract}

Index Terms: Vigna unguiculata, antixenosis, silverleaf whitefly, host plant resistance.

\section{Introdução}

O cultivo do feijão-caupi [Vigna unguiculata (L.) Walp.] é comum em regiões tropicais e subtropicais do mundo, e a espécie é um alimento básico para a população dessas regiões, com elevado valor nutritivo, proteico e energético (Singh et al., 2002). No Brasil, o cultivo do feijão-caupi está se expandindo para a região dos Cerrados, nas regiões Norte, Nordeste e Centro Oeste, onde é explorado na safrinha, após as culturas de soja e arroz, ou como cultura principal, em alguns locais (Freire Filho et al., 2011).

Amosca-branca, Bemisia tabaci (Gennadius) biótipo B (Hemiptera: Aleyrodidae), tem se destacado como uma das mais limitantes pragas da cultura (Silva et al., 2004). Além dos danos diretos ao desenvolvimento vegetativo e reprodutivo das plantas (Musa \& Ren, 2005; Lacerda \& Carvalho, 2008), esse hemíptero é considerado um dos mais importantes vetores de patógenos virais do mundo, pois veicula mais de 110 fitoviroses (Jones, 2003) - princialmente do grupo dos geminivírus. Para o feijão-caupi, B. tabaci biótipo B destaca-se principalmente por sua capacidade de transmitir o vírus do mosaico-dourado do feijão-caupi, que provoca expressivas perdas na produção (Fazolin et al., 2009).

O manejo dessa praga é dificultado em razão de uma série de particularidades apresentadas pelo inseto, tais 
como sua grande capacidade de reprodução e adaptação a condições adversas, ampla gama de hospedeiros e rapidez no desenvolvimento de resistência aos diferentes grupos químicos de inseticidas (Alencar et al., 2004). Em geral, o controle de B. tabaci temse baseado principalmente na aplicação de inseticidas sintéticos de amplo espectro, o que muitas vezes inviabiliza a adoção de outras táticas de controle (Alencar et al., 2004).

O emprego da resistência varietal é considerado um método ideal para o controle de pragas, pela possibilidade de manter a população de insetos abaixo do nível de dano econômico, sem interferir no meio ambiente (Vendramim \& Guzzo, 2009). Além disso, esse método é de fácil utilização, não interfere nas demais práticas culturais e é compatível com outras práticas do manejo integrado de pragas (Painter, 1951; Smith, 2005). Nesse sentido, há pesquisas brasileiras promissoras para obtenção de genótipos de feijão-caupi resistentes a $B$. tabaci biótipo $\mathrm{B}$, com base em testes de preferência quanto à alimentação e oviposição (Costa et al., 2004; Silva et al., 2008; Rodrigues et al., 2012) e testes quanto à antibiose (Rodrigues et al., 2012). No entanto, todos estes trabalhos avaliaram pequeno número de materiais, o que justifica que as investigações nessa área sejam aprofundadas com uso de um maior número de genótipos.

O objetivo deste trabalho foi avaliar a atratividade de 51 genótipos de feijão-caupi para oviposição de Bemisia tabaci biótipo $\mathrm{B}$ e identificar possíveis fontes de resistência à mosca-branca.

\section{Material e Métodos}

O trabalho foi desenvolvido em casa de vegetação $\left(\mathrm{T}=22 \pm 15^{\circ} \mathrm{C}, \mathrm{UR}=65 \pm 10 \%\right.$, fotoperíodo médio de 12 horas) da Faculdade de Ciências Agronômicas, da Universidade Estadual Paulista, Campus de Botucatu $\left(22^{\circ} 85^{\prime} \mathrm{S}, 48^{\circ} 26^{\prime} \mathrm{W}\right)$, de dezembro de 2010 a setembro de 2011. Os 51 genótipos de feijão-caupi (Tabela 1) utilizados foram fornecidos pelo Banco de Germoplasma do Centro de Pesquisa Agropecuária do Meio-Norte (Embrapa Meio-Norte).

A criação de mosca-branca foi feita em gaiolas metálicas $(2,0 \times 2,5 \times 2,0 \mathrm{~m})$, revestidas lateralmente por telado antiafídeo (200 mesh) branco, com teto coberto por plástico e sombrite. Utilizou-se couve-de-folhas (Brassica oleracea var. acephala L.) - plantadas em vasos alocados em bancadas no interior das gaiolas - como fonte de alimentação para os insetos. O monitoramento dos vasos e a troca de plantas deterioradas por sadias foram realizados semanalmente.

Os experimentos foram divididos em duas etapas. $\mathrm{Na}$ primeira, foram realizados testes preliminares com chance de escolha quanto à atratividade e à preferência para oviposição de $B$. tabaci, com os 51 genótipos de feijão-caupi. As plantas utilizadas nos ensaios foram formadas em vasos de $2 \mathrm{~L}$, preenchidos com substrato formado por solo (Latossolo Vermelho-Escuro), areia grossa lavada e matéria orgânica (esterco de curral curtido), na proporção de 1:1:1. A adubação foi realizada no dia da semeadura com $0,33 \mathrm{~g}$ de superfosfato simples e $0,16 \mathrm{~g}$ de cloreto de potássio em cada vaso, com base na análise de solo e nos níveis recomendados para a cultura (Melo et al., 2005). Utilizaram-se três sementes por vaso, para cada um dos genótipos. Quando as plantas estavam com a primeira folha trifoliolada completamente expandida (estádio V3), foi realizado o desbaste, tendo-se deixado uma planta por vaso.

Cinquenta genótipos foram divididos aleatoriamente em dois grupos de 25. O genótipo Canapu foi incluído como testemunha nos dois grupos, uma vez que se trata de material comercial suscetível a $B$. tabaci (Costa et al., 2004).

Antes da infestação, as folhas cotiledonares foram retiradas das plantas e, após dois dias, foram dispostas ao acaso, em círculo, no interior de gaiolas metálicas com 2,5x3,0x2,5 m, teto revestido por plástico e sombrite $(30 \%)$, e laterais revestidas por telado antiafídeo. Os vasos ficaram espaçados em $15 \mathrm{~cm}$, para evitar o contato entre as plantas. Cada gaiola, com 26 vasos, foi considerada uma repetição. Empregou-se o delineamento de blocos ao acaso (DBC), com cinco repetições simultâneas para cada grupo. As gaiolas foram infestadas com cerca de 2.600 adultos de mosca-branca (aproximadamente 1.300 casais), com 48 horas de idade.

Para a obtenção de insetos com essa idade, foram acopladas, 48 horas antes da montagem do experimento, gaiolas aos vasos utilizados na criação, para se ter certeza de que os adultos eram novos e com alto potencial de infestação. As moscas foram coletadas com auxílio de sugador de borracha acoplado a um tubo de vidro de $11 \mathrm{~cm}$ de altura e $4 \mathrm{~cm}$ de diâmetro, e 
Tabela 1. Genótipos de feijão caupi utilizados nas avaliações de resistência à Bemisia tabaci biótipo B, com as respectivas genealogias.

\begin{tabular}{|c|c|}
\hline Genótipo & Genealogia/origem \\
\hline BR 14-Mulato(1) & $\mathrm{CNC} 0434 \mathrm{X} \mathrm{CNCx} 27-2 \mathrm{E}$ \\
\hline BR 17 Gurgueia $^{(1)}$ & BR10 - Piauí X CE 315 \\
\hline BR 3-Tracuateua ${ }^{(1)}$ & Selecionada na cultivar local Quebra cadeira ou Cheque ouro: procedente da região Nordeste do Brasil \\
\hline BRS-Cauamé ${ }^{(1)}$ & TE93 $21013 \mathrm{~F}$ X TE96 $28222 \mathrm{G}$ \\
\hline BRS-Milênio ${ }^{(1)}$ & Selecionada na cultivar BR3 Tracuateua \\
\hline BRS-Novaera $^{(1)}$ & TE97 - $404-1$ F X TE97 $404-3 F$ \\
\hline BRS-Pajeú ${ }^{(1)}$ & CNCx405 - 17F X TE94 268 3D \\
\hline BRS-Paraguaçu $^{(1)}$ & BR10 - Piauí X Aparecido Moita \\
\hline BRS-Potengi ${ }^{(1)}$ & TE96 282 22E X TE93 210 13F \\
\hline BRS-Rouxinol $^{(1)}$ & TE86 75 57E X TEx1 69E \\
\hline BRS-Tumucumaqui $^{(1)}$ & TE96 282 22G X IT87 D 6113 \\
\hline BRS-Urubuquara $^{(1)}$ & Selecionada na cultivar BR3 Tracuateua \\
\hline BRS-Marataoã $\tilde{a}^{(1)}$ & Seridó X TVx 1836 013J \\
\hline Canapu (padrão) $)^{(1)}$ & Cultivar local São Julião, Piauí \\
\hline Canapuzinho $^{(1)}$ & Cultivar local São Raimundo Nonato, Piauí \\
\hline Canapuzinho $1-2^{(1)}$ & Selecionada na cultivar Canapuzinho, São Raimundo Nonato, Piauí \\
\hline Capela $^{(1)}$ & Cultivar local, Capela do Alto, São Paulo \\
\hline Corujinha $^{(1)}$ & Cultivar local Barbalha, Ceará \\
\hline Epace $10^{(1)}$ & Seridó x TVu 1888 \\
\hline Inhuma $^{(1)}$ & Selecionada na cultivar local Inhuma, Inhuma, Piauí \\
\hline Monteiro $^{(1)}$ & Selecionada na cultivar local Monteiro, Piripiri, Piauí \\
\hline Patativa $^{(1)}$ & CNC1735 X (CNCx926 4F X Paulista) \\
\hline Paulistinha $^{(1)}$ & Cultivar local, Barbalha, Ceará \\
\hline Pingo-de-ouro-1-1 ${ }^{(1)}$ & Selecionada na cultivar local Pingo de ouro, Iguatu, Ceará \\
\hline Poços-de-Caldas - $\mathrm{MG}^{(1)}$ & Selecionada em cultivar introduzida de triagem indefinida do Peru, provavelmente cultivar Vainablanca \\
\hline Sanzi Sambili(1) & International Institute of Tropical Agriculture (IITA), Ibadan, Nigéria \\
\hline Vainablanca $^{(1)}$ & Cultivar melhorada oriunda do Peru \\
\hline IT81 D-1045 Enramador ${ }^{(2)}$ & International Institute of Tropical Agriculture (IITA), Ibadan, Nigéria \\
\hline IT81 D-1045 Ereto ${ }^{(2)}$ & International Institute of Tropical Agriculture (IITA), Ibadan, Nigéria \\
\hline IT82 D-889 (2) & International Institute of Tropical Agriculture (IITA), Ibadan, Nigéria \\
\hline IT85 F-2687(2) & International Institute of Tropical Agriculture (IITA), Ibadan, Nigéria \\
\hline IT86 D-716-1 (2) & International Institute of Tropical Agriculture (IITA), Ibadan, Nigéria \\
\hline MN05-841-B 49(2) & MNC00 599 F 9 X MNC99 537F 142 \\
\hline MNC04-786-B - $872^{(2)}$ & MNCOI 625E 10125 X MNC99554 D 10122 \\
\hline MNC99-505 G-11 (2) & Canapuzinho X BR17 Gurgueia \\
\hline MNC99-507 G-4(2) & BR14 Mulato X Canapuzinho \\
\hline MNC99-507 G-8 ${ }^{(2)}$ & BR14 Mulato X Canapuzinho \\
\hline MNC99-508-1 $1^{(2)}$ & TE90 180 88F X Canapuzinho \\
\hline MNC99-510 G-16 (2) & Paulista X TE90 180 88F \\
\hline MNC99-541 F-15(2) & TE93 $21013 \mathrm{~F}$ X TE96 $28222 \mathrm{G}$ \\
\hline MNC99-541 F-21 (2) & TE93 21013 F X TE96 $28222 \mathrm{G}$ \\
\hline TE93 - 244-23 F-1(2) & IPA 206 X TE86 73 3G \\
\hline TE94 - 309 G-9(2) & CNCx405 24F X CNCx698 128G \\
\hline TE97 - 299 G-24(2) & CNCx405 17F X CNCx698 128G \\
\hline TE97 - 304 G-4(2) & CNCx405 17F X TE94 268 3D \\
\hline TE97 - 309 G-18(2) & CNCx405 24F X CNCx698 128G \\
\hline TE97 - 309 G-24(2) & CNCx405 24F X CNCx698 128G \\
\hline TVU-1593(2) & International Institute of Tropical Agriculture (IITA), Ibadan, Nigéria \\
\hline TVU-36 & International Institute of Tropical Agriculture (IITA), Ibadan, Nigéria \\
\hline TVU-382(2) & International Institute of Tropical Agriculture (IITA), Ibadan, Nigéria \\
\hline
\end{tabular}

${ }^{(1)}$ Cultivar. ${ }^{(2)}$ Linhagem. CNC, Centro Nacional Caupi; MNC, Meio-Norte Caupi. 
liberadas no piso e no centro das gaiolas. Na coleta dos insetos, deu-se preferência aos pares de mosca-branca, uma vez que, segundo Byrne \& Bellows Junior (1991), os casais do inseto costumam ficar pareados.

A atratividade foi avaliada 24, 48 e 72 horas após a liberação dos insetos, tendo-se contado, com o auxílio de um espelho, o número de adultos presentes na parte abaxial dos folíolos. Após a última avaliação, retirou-se o trifólio de cada planta e, no laboratório, contou-se o número de ovos presentes na face abaxial (Baldin et al., 2005), com o auxílio de microscópio estereoscópico (aumento de 40X). Após a contagem, foi medida a área foliar, com auxílio de medidor foliar LI 3000A (LI-COR Inc., Lincoln, NE, EUA), para determinação do número de ovos por $\mathrm{cm}^{2}$.

Com base nos resultados desse teste preliminar com chance de escolha, foram selecionados 14 genótipos, dos quais dez (cinco de cada grupo) apresentaram os menores valores médios de ovos por $\mathrm{cm}^{2}-$ portanto, promissores quanto à resistência -, três apresentaram as maiores médias de oviposição (suscetíveis), além do genótipo Canapu, mantido como padrão comercial suscetível. A metodologia utilizada para a avaliação da atratividade e da preferência para oviposição com chance de escolha foi a mesma descrita no teste preliminar. Adotou-se o delineamento de blocos ao acaso, com cinco repetições (uma gaiola representou uma repetição).

A oviposição também foi avaliada em teste sem chance de escolha, com os 14 genótipos de feijão-caupi. As plantas (estádio V3) foram individualizadas em gaiolas metálicas (vazadas), revestidas com tecido "voil", nas quais foram liberados 50 casais de mosca-branca (48 horas de idade). Após 72 horas de infestação, foi realizada a contagem dos ovos, conforme descrito para o teste preliminar. Nesse caso, adotou-se o delineamento experimental inteiramente casualizado, com seis repetições (cada planta individualizada representou uma repetição).

Para os 14 genótipos, o índice de preferência para oviposição foi obtido pela fórmula, $\mathrm{IPO}=[(\mathrm{T}-\mathrm{P}) /(\mathrm{T}+\mathrm{P})] \times 100$, em que: T é o número de ovos contados no tratamento e $\mathrm{P}$ é o número de ovos contados no genótipo-padrão suscetível Canapu. $\mathrm{O}$ índice varia de +100 (muito estimulante) até -100 (total deterrência), e o valor 0 indica neutralidade. A classificação foi feita a partir da comparação das médias de ovos dos tratamentos com a média do tratamento-padrão, tendo-se levado em consideração o erro-padrão da média (Fenemore, 1980; Baldin et al., 2005; Schilick-Souza et al., 2011).

Os dados obtidos foram submetidos à análise de variância pelo teste F. Verificou-se a normalidade dos dados por meio do teste de Shapiro-Wilk, e a homogeneidade pelo teste de Levene. Utilizaramse as transformações $(\mathrm{x}+0,5)^{1 / 2}$ e arcosseno de $(\mathrm{x}+0,5)^{1 / 2}$, quando os dados não apresentavam distribuição normal e homogeneidade entre as variâncias (Banzatto \& Kronka, 2006).

Quando o teste $\mathrm{F}$ apresentou significância, as médias foram comparadas pelos testes de Tukey e de Scott- Knott, a 5\% de probabilidade, com uso do programa estatístico Sisvar 5.0. Os dados referentes à atratividade de adultos e o número de ovos por $\mathrm{cm}^{2}$ foram transformados em $(x+0,5)^{1 / 2}$.

\section{Resultados e Discussão}

Foram verificadas diferenças significativas entre os 26 genótipos de feijão-caupi avaliados no primeiro grupo (cultivar Canapu mais 25 outros), quanto à atratividade de $B$. tabaci biótipo $\mathrm{B}$, nas três avaliações do teste preliminar (24, 48 e $72 \mathrm{~h}$ após a liberação dos insetos, Tabela 2). Considerando-se as médias gerais, os genótipos TE93-244-23-F1, MNC99-541-F21, Epace 10 e MNC04-786-B87-2 foram os menos atrativos, enquanto os genótipos Pingo-de-ouro-1-1 e TVU 1593 atraíram o maior número de insetos. Os genótipos Pingo-de-ouro 1-1, Poços de Caldas, Inhuma, IT81 D-1045 Ereto, TVU-1593, Canapuzinho 1-2 e MNC99-510 G-16 foram os mais ovipositados e diferiram dos demais materiais. Entre os menos ovipositados, TE93-244-23 F-1, Patativa, BRS-Marataoã, MNC99-541-F-21 e TE94 309 G-9 destacaram-se com as menores médias de ovos por $\mathrm{cm}^{2}$, e foram selecionados para a segunda etapa de ensaios.

Entre os genótipos do segundo grupo avaliado no teste preliminar, também foram observadas diferenças significativas entre os genótipos durante as três avaliações (Tabela 3). TVU-36 e BRS-Urubuquara foram os menos atrativos e diferiram dos demais materiais. Os genótipos MN05-841 B-49, IT81 D-1045 Enramador, Canapu, Vainablanca, MNC99-510-8 e MNC99-507 G-8 foram os mais atrativos. Quanto à oviposição, os genótipos TVU-36, BR 17-Gurgueia, TE97-304 G-4, BRSUrubuquara e BRS-Rouxinol destacaram-se com médias inferiores a 2 ovos por $\mathrm{cm}^{2}$ - o que indica a ocorrência de não preferência para oviposição - e foram selecionados 
para os ensaios subsequentes. Rodrigues et al. (2012) verificaram que BRS-Urubuquara apresenta deterrência quanto à oviposição de $B$. tabaci biótipo $\mathrm{B}$, em teste com chance de escolha, o que é corroborado pelos resultados do presente trabalho e indica uma importante fonte de resistência a ser utilizada em programas de melhoramento.

No ensaio de atratividade com os 14 genótipos de feijão-caupi pré-selecionados, também foram verificadas diferenças entre os materiais nas três avaliações (Tabela 4). Após 24 horas da liberação dos adultos, TE97-304 G-4, MNC99-541 F-21, BRS-Marataoã, BR 17-Gurgueia, TE93-244 23-F1, TVU-36 e BRS-Urubuquara foram os menos atrativos e diferiram de Patativa. Os demais genótipos apresentaram valores intermediários. Os materiais menos atrativos no período de 24 horas, mantiveram esse comportamento nas avaliações às 48 e 72 horas. Patativa, TVU-1593 e Pingo-de-ouro-1-1 foram os mais atrativos a $B$. tabaci.

Ao se considerar o número médio geral de adultos de mosca-branca atraídos, os genótipos Patativa, Pingo-de-ouro-1-1 e TVU-1593 foram os mais atrativos ao inseto (Tabela 4). Pingo-de-ouro-1-1 e TVU-1593 mantiveram o mesmo comportamento apresentado no primeiro ensaio, em que se destacaram entre os mais atrativos. Isso indica uma maior volatilização de compostos atraentes nestes genótipos. De maneira oposta, BRS-Urubuquara, BRS-Marataoã, MNC99-541 F-21, BR 17-Gurgueia, TE93-244 23-F1, TVU-36 destacaram-se pela baixa atratividade aos insetos, o que indica a ocorrência de não preferência como mecanismo de resistência à $B$. tabaci.

Tabela 2. Média \pm erro padrão do número de insetos atraídos e de ovos de Bemisia tabaci biótipo B sobre os 26 genótipos de feijão-caupi do primeiro grupo (25 genótipos além da testemunha Canapu), em ensaio com chance de escolha, em casa de vegetação ${ }^{(1)}$.

\begin{tabular}{|c|c|c|c|c|c|}
\hline \multirow[t]{2}{*}{ Genótipo } & \multicolumn{3}{|c|}{ Número de insetos atraídos } & \multirow[t]{2}{*}{ Média geral ${ }^{(2)}$} & \multirow{2}{*}{$\begin{array}{l}\text { Número de ovos } \\
\text { por } \mathrm{cm}^{2}\end{array}$} \\
\hline & $24 \mathrm{~h}$ & $48 \mathrm{~h}$ & $72 \mathrm{~h}$ & & \\
\hline Pingo-de-ouro-1-1 & $194,0 \pm 63,43 a$ & $220,2 \pm 72,46 a$ & $229,4 \pm 77,76 a$ & $214,5 \pm 10,60 \mathrm{a}$ & $25,2 \pm 6,84 \mathrm{a}$ \\
\hline TVU-1593 & $125,4 \pm 24,23 \mathrm{a}$ & $253,8 \pm 45,59 a$ & $228,2 \pm 44,60 \mathrm{a}$ & $202,5 \pm 39,24 a$ & $24,6 \pm 9,97 \mathrm{a}$ \\
\hline Poços de Caldas & $169,0 \pm 63,31 \mathrm{a}$ & $168,8 \pm 88,12 \mathrm{a}$ & $170,2 \pm 97,24 a$ & $169,3 \pm 0,44 b$ & $20,6 \pm 7,54 a$ \\
\hline Inhuma & $160,0 \pm 65,91 \mathrm{a}$ & $179,8 \pm 68,51 \mathrm{a}$ & $160,2 \pm 49,45 \mathrm{a}$ & $166,7 \pm 6,57 \mathrm{~b}$ & $27,7 \pm 13,85 \mathrm{a}$ \\
\hline Canapuzinho 1-2 & $124,6 \pm 35,92 \mathrm{a}$ & $155,8 \pm 59,24 a$ & $182,4 \pm 67,76 a$ & $154,3 \pm 16,70 \mathrm{c}$ & $22,2 \pm 16,87 \mathrm{a}$ \\
\hline IT81 D-1045 Ereto & $147,8 \pm 51,90 \mathrm{a}$ & $150,8 \pm 39,31 \mathrm{a}$ & $144,6 \pm 39,89 a$ & $147,7 \pm 1,79 \mathrm{c}$ & $30,5 \pm 16,79 \mathrm{a}$ \\
\hline MNC99-510 G16 & $94,8 \pm 56,96 \mathrm{a}$ & $147,4 \pm 66,92 \mathrm{a}$ & $143,6 \pm 76,67 a$ & $128,6 \pm 16,94 \mathrm{c}$ & $20,4 \pm 10,31 \mathrm{a}$ \\
\hline BRS-Novaera & $78,4 \pm 45,40 \mathrm{~b}$ & $117,4 \pm 46,96 \mathrm{a}$ & $112,0 \pm 52,25 \mathrm{a}$ & $102,6 \pm 12,20 \mathrm{~d}$ & $7,8 \pm 2,52 b$ \\
\hline IT85 F-2687 & $71,2 \pm 33,97 \mathrm{~b}$ & $97,0 \pm 76,64 b$ & $83,0 \pm 45,94 \mathrm{~b}$ & $83,7 \pm 7,46 \mathrm{e}$ & $6,6 \pm 3,31 b$ \\
\hline TE97-299 G-24 & $93,6 \pm 26,77 \mathrm{a}$ & $71,2 \pm 19,17 \mathrm{~b}$ & $73,4 \pm 22,73 b$ & $79,4 \pm 7,13 \mathrm{e}$ & $3,5 \pm 1,10 \mathrm{~b}$ \\
\hline Monteiro & $84,6 \pm 26,60 \mathrm{a}$ & $79,0 \pm 10,32 b$ & $74,4 \pm 22,69 b$ & $79,3 \pm 2,95 \mathrm{e}$ & $5,3 \pm 2,47 b$ \\
\hline Canapu & $86,0 \pm 26,22 \mathrm{a}$ & $83,4 \pm 23,56 b$ & $57,2 \pm 16,62 b$ & $75,5 \pm 9,20 \mathrm{e}$ & $7,9 \pm 2,26 b$ \\
\hline Paulistinha & $64,2 \pm 16,62 b$ & $71,6 \pm 21,72 b$ & $82,2 \pm 25,75 b$ & $72,7 \pm 5,22 \mathrm{e}$ & $3,9 \pm 1,95 b$ \\
\hline BRS-Paraguaçu & $65,6 \pm 25,34 \mathrm{~b}$ & $70,2 \pm 34,16 b$ & $52,4 \pm 23,60 \mathrm{~b}$ & $62,6 \pm 5,30 \mathrm{f}$ & $9,0 \pm 7,12 b$ \\
\hline MNC99-508-1 & $59,4 \pm 25,50 \mathrm{~b}$ & $54,2 \pm 25,10 \mathrm{~b}$ & $57,4 \pm 20,05 b$ & $57,0 \pm 1,51 \mathrm{f}$ & $5,1 \pm 1,62 b$ \\
\hline BRS-Tumucumaqui & $40,8 \pm 18,88 \mathrm{~b}$ & $61,4 \pm 39,44 b$ & $61,8 \pm 35,83 b$ & $54,7 \pm 6,93 \mathrm{f}$ & $5,9 \pm 2,24 b$ \\
\hline MNC99-541-F-15 & $58,4 \pm 21,51 \mathrm{~b}$ & $49,2 \pm 18,91 b$ & $46,2 \pm 20,92 b$ & $51,3 \pm 3,67 f$ & $6,7 \pm 3,75 b$ \\
\hline Corujinha & $50,2 \pm 16,95 \mathrm{~b}$ & $34,6 \pm 10,79 b$ & $34,6 \pm 15,87 \mathrm{~b}$ & $39,8 \pm 5,20 \mathrm{~g}$ & $7,9 \pm 3,31 b$ \\
\hline IT86 D-716-1 & $49,2 \pm 16,78 b$ & $31,4 \pm 6,66 b$ & $29,4 \pm 7,85 b$ & $36,7 \pm 6,29 \mathrm{~g}$ & $4,0 \pm 0,81 b$ \\
\hline TE94 309 G-9 & $47,4 \pm 24,96 \mathrm{~b}$ & $35,6 \pm 19,09 b$ & $25,8 \pm 13,17 \mathrm{~b}$ & $36,3 \pm 6,24 \mathrm{~g}$ & $2,3 \pm 0,94 b$ \\
\hline Patativa & $36,6 \pm 9,14 b$ & $31,0 \pm 6,44 b$ & $28,8 \pm 5,39 b$ & $32,1 \pm 2,26 \mathrm{~g}$ & $1,5 \pm 0,43 b$ \\
\hline BRS-Marataoã & $34,2 \pm 8,87 \mathrm{~b}$ & $29,2 \pm 8,71 b$ & $27,2 \pm 6,57 b$ & $30,2 \pm 2,08 \mathrm{~g}$ & $1,7 \pm 0,59 b$ \\
\hline MNC04-786-B87-2 & $28,6 \pm 6,18 b$ & $22,0 \pm 4,52 b$ & $21,4 \pm 3,68 b$ & $24,0 \pm 2,31 \mathrm{~h}$ & $4,9 \pm 2,24 b$ \\
\hline Epace 10 & $25,6 \pm 10,25 b$ & $24,8 \pm 9,04 b$ & $23,2 \pm 10,30 \mathrm{~b}$ & $24,5 \pm 0,71 \mathrm{~h}$ & $5,1 \pm 2,27 b$ \\
\hline MNC99-541-F-21 & $27,6 \pm 17,67 \mathrm{~b}$ & $24,4 \pm 16,21 b$ & $18,0 \pm 12,27 \mathrm{~b}$ & $22,7 \pm 3,39 h$ & $2,2 \pm 0,91 b$ \\
\hline TE93-244-23 F-1 & $19,6 \pm 3,48 b$ & $11,6 \pm 2,94 \mathrm{~b}$ & $10,6 \pm 1,53 b$ & $13,9 \pm 2,85 \mathrm{~h}$ & $1,2 \pm 0,43 \mathrm{~b}$ \\
\hline $\mathrm{F}$ & $2,54^{*}$ & $3,90 *$ & $1,80^{*}$ & $42,63 *$ & $2,04 *$ \\
\hline CV (\%) & 37,06 & 44,72 & 52,62 & 9,73 & 53,14 \\
\hline
\end{tabular}

(1)Médias seguidas de letras iguais, nas colunas, não diferem entre si, pelo teste de Scott-Knott, a $5 \%$ de probabilidade. Dados originais para análise foram transformados em $(x+0,5)^{1 / 2} .{ }^{(2)}$ Média do número de insetos atraídos nas três avaliações (24, 48 e 72 horas). 
Tabela 3. Média \pm erro padrão do número de insetos atraídos e de ovos de Bemisia tabaci biótipo B sobre os 26 genótipos de feijão-caupi do segundo grupo ( 25 genótipos além da testemunha Canapu), em ensaio com chance de escolha, em casa de vegetação(1).

\begin{tabular}{|c|c|c|c|c|c|}
\hline \multirow[t]{2}{*}{ Genótipo } & \multicolumn{3}{|c|}{ Número de insetos atraídos } & \multirow[t]{2}{*}{ Média geral $^{(2)}$} & \multirow{2}{*}{$\begin{array}{l}\text { Número de ovos } \\
\text { por } \mathrm{cm}^{2}\end{array}$} \\
\hline & $24 \mathrm{~h}$ & $48 \mathrm{~h}$ & $72 \mathrm{~h}$ & & \\
\hline MN05-841 B-49 & $78,2 \pm 35,05 \mathrm{a}$ & $154,5 \pm 100,30 \mathrm{a}$ & $144,7 \pm 88,13 \mathrm{a}$ & $125,8 \pm 23,96 a$ & $15,2 \pm 2,07 \mathrm{a}$ \\
\hline IT81 D-1045 Enramador & $94,5 \pm 45,85 \mathrm{a}$ & $118,5 \pm 53,89 \mathrm{a}$ & $97,5 \pm 41,04 \mathrm{a}$ & $103,5 \pm 7,55 \mathrm{a}$ & $20,4 \pm 10,86 a$ \\
\hline Canapu & $93,5 \pm 34,64 a$ & $94,2 \pm 46,87 \mathrm{a}$ & $95,5 \pm 39,37 \mathrm{a}$ & $94,4 \pm 0,58 \mathrm{a}$ & $7,7 \pm 3,14 \mathrm{a}$ \\
\hline Vainablanca & $102,2 \pm 29,23 \mathrm{a}$ & $82,0 \pm 36,40 \mathrm{a}$ & $85,7 \pm 39,52 \mathrm{a}$ & $90,0 \pm 6,22 \mathrm{a}$ & $12,6 \pm 3,60 \mathrm{a}$ \\
\hline MNC99-510-8 & $56,5 \pm 28,31 \mathrm{a}$ & $101,7 \pm 72,05 \mathrm{a}$ & $107,5 \pm 80,41 \mathrm{a}$ & $88,5 \pm 16,13 \mathrm{a}$ & $15,2 \pm 12,56 \mathrm{a}$ \\
\hline MNC99-507 G8 & $64,7 \pm 23,53 \mathrm{a}$ & $96,2 \pm 50,31 \mathrm{a}$ & $94,0 \pm 43,90 \mathrm{a}$ & $85,0 \pm 10,15 \mathrm{a}$ & $11,2 \pm 4,38 \mathrm{a}$ \\
\hline BRS Milênio & $77,7 \pm 26,30 \mathrm{a}$ & $72,5 \pm 33,02 \mathrm{a}$ & $72,5 \pm 30,66 \mathrm{a}$ & $74,2 \pm 1,75 b$ & $7,7 \pm 5,84 \mathrm{a}$ \\
\hline MNC99-507 G4 & $76,0 \pm 25,77 \mathrm{a}$ & $93,7 \pm 35,31 \mathrm{a}$ & $53,0 \pm 19,76 \mathrm{a}$ & $74,2 \pm 11,80 \mathrm{~b}$ & $4,5 \pm 1,71 \mathrm{~b}$ \\
\hline BR3-Tracuateua & $87,5 \pm 42,96 a$ & $67,5 \pm 32,92 \mathrm{a}$ & $65,5 \pm 45,33 \mathrm{a}$ & $73,5 \pm 7,02 \mathrm{~b}$ & $3,8 \pm 1,92 b$ \\
\hline TE97-309 G-24 & $80,2 \pm 37,63 \mathrm{a}$ & $89,7 \pm 53,41 \mathrm{a}$ & $38,5 \pm 19,68 b$ & $69,5 \pm 15,74 b$ & $6,3 \pm 2,67 \mathrm{a}$ \\
\hline BR 14-Mulato & $62,7 \pm 20,21 \mathrm{a}$ & $73,0 \pm 41,65 \mathrm{a}$ & $56,7 \pm 26,67 \mathrm{a}$ & $64,1 \pm 4,74 \mathrm{~b}$ & $7,6 \pm 5,01 \mathrm{a}$ \\
\hline TE97-309 G 18 & $68,5 \pm 25,62 \mathrm{a}$ & $65,7 \pm 23,87 \mathrm{a}$ & $39,7 \pm 13,51 b$ & $58,0 \pm 9,16 \mathrm{~b}$ & $2,5 \pm 0,54 \mathrm{~b}$ \\
\hline MNC99-505 G11 & $48,0 \pm 12,19 \mathrm{a}$ & $38,0 \pm 19,91 \mathrm{~b}$ & $30,2 \pm 18,05 b$ & $38,7 \pm 5,14 \mathrm{c}$ & $3,4 \pm 0,95 b$ \\
\hline Sanzi Sambili & $35,2 \pm 18,15 b$ & $36,2 \pm 15,04 b$ & $42,7 \pm 20,84 b$ & $38,0 \pm 2,35 \mathrm{c}$ & $2,6 \pm 1,53 b$ \\
\hline BRS-Pajeú & $41,0 \pm 11,73 b$ & $30,2 \pm 9,52 b$ & $42,7 \pm 14,34 b$ & $38,0 \pm 3,91 \mathrm{c}$ & $5,2 \pm 2,31 \mathrm{~b}$ \\
\hline Canapuzinho & $33,2 \pm 11,23 b$ & $37,0 \pm 9,61 b$ & $43,2 \pm 19,44 \mathrm{~b}$ & $37,8 \pm 2,92 \mathrm{c}$ & $4,3 \pm 2,07 \mathrm{~b}$ \\
\hline BRS-Cauamé & $55,0 \pm 31,13 \mathrm{a}$ & $20,5 \pm 3,75 b$ & $22,7 \pm 6,61 b$ & $32,7 \pm 11,14 \mathrm{c}$ & $2,2 \pm 0,48 \mathrm{~b}$ \\
\hline TVU-382 & $38,7 \pm 10,33 b$ & $28,0 \pm 9,76 b$ & $22,5 \pm 10,42 b$ & $29,7 \pm 4,77 \mathrm{c}$ & $2,0 \pm 0,52 b$ \\
\hline Capela & $34,7 \pm 15,68 \mathrm{~b}$ & $27,7 \pm 10,54 b$ & $25,5 \pm 8,34 \mathrm{~b}$ & $29,3 \pm 2,79 \mathrm{c}$ & $3,2 \pm 0,59 \mathrm{~b}$ \\
\hline BRS-Rouxinol & $35,0 \pm 10,10 \mathrm{~b}$ & $26,2 \pm 15,99 \mathrm{~b}$ & $13,2 \pm 4,64 \mathrm{~b}$ & $24,8 \pm 6,32 d$ & $1,5 \pm 0,58 \mathrm{~b}$ \\
\hline BRS-Potengi & $25,7 \pm 10,37 \mathrm{~b}$ & $22,0 \pm 13,08 \mathrm{~b}$ & $20,2 \pm 7,19 \mathrm{~b}$ & $22,6 \pm 1,62 d$ & $4,8 \pm 1,06 \mathrm{~b}$ \\
\hline BR 17-Gurgueia & $27,5 \pm 9,54 \mathrm{~b}$ & $23,0 \pm 12,00 \mathrm{~b}$ & $16,5 \pm 5,33 b$ & $22,3 \pm 3,19 \mathrm{~d}$ & $0,7 \pm 0,47 \mathrm{~b}$ \\
\hline TE97-304 G 4 & $21,7 \pm 8,10 \mathrm{~b}$ & $20,0 \pm 6,72 b$ & $16,7 \pm 9,26 \mathrm{~b}$ & $19,5 \pm 1,46 \mathrm{~d}$ & $0,9 \pm 0,39 \mathrm{~b}$ \\
\hline IT82 D-889 & $26,2 \pm 6,79 \mathrm{~b}$ & $12,7 \pm 4,38 b$ & $14,5 \pm 4,03 b$ & $17,8 \pm 4,24 \mathrm{~d}$ & $2,6 \pm 0,83 b$ \\
\hline TVU-36 & $15,0 \pm 7,32 \mathrm{~b}$ & $11,2 \pm 2,83 \mathrm{~b}$ & $12,2 \pm 5,45 \mathrm{~b}$ & $12,8 \pm 1,12 \mathrm{e}$ & $0,5 \pm 0,28 \mathrm{~b}$ \\
\hline BRS-Urubuquara & $6,2 \pm 0,62 \mathrm{~b}$ & $6,5 \pm 1,65 \mathrm{~b}$ & $6,75 \pm 2,46 \mathrm{~b}$ & $6,5 \pm 0,14 \mathrm{e}$ & $1,4 \pm 0,71 \mathrm{~b}$ \\
\hline F & $3,11^{*}$ & $3,90^{*}$ & $3,83^{*}$ & $19,74^{*}$ & $3,11 *$ \\
\hline CV (\%) & 38,62 & 44,72 & 46,01 & 12,84 & 51,95 \\
\hline
\end{tabular}

${ }^{(1)}$ Médias seguidas de letras iguais, nas colunas, não diferem entre si, pelo teste de Scott-Knott, a $5 \%$ de probabilidade. Dados originais para análise foram transformados em $(x+0,5)^{1 / 2} \cdot{ }^{(2)}$ Média do número de insetos atraídos nas três avaliações (24, 48 e 72 horas).

Tabela 4. Média \pm erro padrão do número de adultos de Bemisia tabaci biótipo B atraídos para genótipos de feijão-caupi, em ensaio com chance de escolha, em casa de vegetação ${ }^{(1)}$.

\begin{tabular}{|c|c|c|c|c|}
\hline \multirow[t]{2}{*}{ Genótipo } & \multicolumn{3}{|c|}{ Número de insetos atraídos } & \multirow[t]{2}{*}{ Média geral ${ }^{(2)}$} \\
\hline & $24 \mathrm{~h}$ & $48 \mathrm{~h}$ & $72 \mathrm{~h}$ & \\
\hline Patativa & $124,0 \pm 43,95 \mathrm{a}$ & $130,8 \pm 39,49 a$ & $138,0 \pm 47,23 \mathrm{a}$ & $130,7 \pm 4,06 \mathrm{a}$ \\
\hline TVU- 1593 & $107,6 \pm 21,16 \mathrm{ab}$ & $147,6 \pm 33,55 \mathrm{a}$ & $133,8 \pm 32,10 \mathrm{a}$ & $129,7 \pm 11,73 \mathrm{a}$ \\
\hline Pingo-de-ouro-1-1 & $96,8 \pm 8,00 \mathrm{abc}$ & $127,0 \pm 12,16 \mathrm{a}$ & $126,6 \pm 13,09 \mathrm{a}$ & $116,8 \pm 10,00 \mathrm{ab}$ \\
\hline Canapu & $106,8 \pm 30,83 \mathrm{ab}$ & $84,0 \pm 41,74 a b$ & $66,8 \pm 26,91 \mathrm{ab}$ & $85,9 \pm 11,58 b c$ \\
\hline TE94-309 G-9 & $74,2 \pm 38,21 \mathrm{abcd}$ & $64,6 \pm 38,61 \mathrm{ab}$ & $64,8 \pm 43,25 \mathrm{ab}$ & $67,9 \pm 3,17 \mathrm{c}$ \\
\hline IT81 D-1045 Enramador & $64,6 \pm 9,72 \mathrm{abcd}$ & $61,0 \pm 13,69 \mathrm{ab}$ & $42,2 \pm 10,66 \mathrm{ab}$ & $55,9 \pm 6,94 \mathrm{~cd}$ \\
\hline BRS-Rouxinol & $34,6 \pm 10,84$ abcde & $40,6 \pm 15,36 \mathrm{ab}$ & $35,4 \pm 15,73 \mathrm{ab}$ & $36,9 \pm 1,88 \mathrm{de}$ \\
\hline TE97-304 G-4 & $24,0 \pm 3,06 \mathrm{bcde}$ & $11,6 \pm 2,83 \mathrm{~b}$ & $14,6 \pm 2,89 \mathrm{~b}$ & $16,7 \pm 3,74 \mathrm{ef}$ \\
\hline MNC99-541 F-21 & $20,4 \pm 5,00 \mathrm{de}$ & $11,6 \pm 3,32 b$ & $8,8 \pm 1,77 \mathrm{~b}$ & $13,6 \pm 3,49 \mathrm{fg}$ \\
\hline BRS-Marataoã & $22,2 \pm 5,03 \mathrm{cde}$ & $9,6 \pm 2,37 b$ & $7,2 \pm 1,24 \mathrm{~b}$ & $13,0 \pm 4,65 \mathrm{fg}$ \\
\hline BR 17-Gurgueia & $15,4 \pm 4,22 \mathrm{de}$ & $8,0 \pm 1,67 b$ & $8,6 \pm 3,58 b$ & $10,7 \pm 2,37 \mathrm{fg}$ \\
\hline TE93-244 23-F1 & $15,2 \pm 5,78 \mathrm{de}$ & $7,6 \pm 1,36 b$ & $3,6 \pm 1,02 b$ & $8,8 \pm 3,40 \mathrm{fg}$ \\
\hline TVU-36 & $10,4 \pm 3,34 \mathrm{de}$ & $5,4 \pm 0,87 \mathrm{~b}$ & $5,8 \pm 1,35 \mathrm{~b}$ & $7,2 \pm 1,60 \mathrm{fg}$ \\
\hline BRS-Urubuquara & $4,2 \pm 1,15 \mathrm{e}$ & $2,6 \pm 0,74 \mathrm{~b}$ & $2,4 \pm 0,50 \mathrm{~b}$ & $3,0 \pm 0,57 \mathrm{~g}$ \\
\hline $\mathrm{F}$ & $7,63^{*}$ & $8,07^{*}$ & $8,50^{*}$ & $81,73^{*}$ \\
\hline $\mathrm{CV}(\%)$ & 37,74 & 47,99 & 49,06 & 10,75 \\
\hline
\end{tabular}

${ }^{(1)}$ Médias seguidas de letras iguais, nas colunas, não diferem entre si, pelo teste de Scott-Knott, a $5 \%$ de probabilidade. Dados originais para análise foram transformados em $(x+0,5)^{1 / 2}$. ${ }^{(2)}$ Médias de insetos atraídos, considerando-se as três avaliações $(24,48$ e 72 horas). 
A preferência da mosca-branca por determinados genótipos está relacionada à volatilização de substâncias químicas das folhas e a fatores físicos relativos às cores das folhas, que podem afetar a seleção hospedeira, tanto para a alimentação quanto para a oviposição do inseto (Lara, 1991; Vendramim \& Guzzo, 2009). Em feijão-caupi, no entanto, estes fatores ainda não foram investigados.

No caso do feijão-caupi, a repelência à $B$. tabaci biótipo B seria muito desejável, pois evitaria a transmissão do vírus do mosaico-dourado e a consequente queda da produtividade. Oliveira et al. (2012) relatam que BR 17-Gurgueia destaca-se como pouco atrativo à mosca-branca e apresenta boa reação às infecções causadas pelo vírus, além de ter sementes com padrão comercial. Os autores sugerem, portanto, que o genótipo seja empregado em programas de melhoramento.

No teste com chance de escolha, os genótipos TE93-244-23 F-1, TVU-36, BRS-Urubuquara e TE97-304 G-4 receberam menor oviposição do que TVU-1593, Pingo-de-ouro-1-1 e IT81 D-1045 Enramador (Tabela 5). BRS-Rouxinol, BRS-Marataoã, MNC99-541 F-21 e BR 17-Gurgueia apresentaram baixa oviposição, tendo diferido apenas de TVU-1593 e Pingo-de-ouro-1-1. Quanto aos índices de preferência para oviposição, no teste com chance de escolha, TVU-1593, Pingo-de-ouro-1-1 e IT81 D-1045 Enramador foram estimulantes em relação ao padrão suscetível Canapu. Patativa e TE94-309 G-9 mostraram-se semelhantes ao Canapu, enquanto os demais genótipos foram deterrentes, comparativamente ao Canapu (Tabela 5).

No teste sem chance de escolha, TVU-36 apresentou a menor média de ovos $\mathrm{cm}^{-2}$ e diferiu de Pingo-de-ouro-1-1, IT81 D-1045 Enramador, TVU 1593 e Canapu, o que indica a ocorrência de não preferência para oviposição (Tabela 5). TE93-244-23 F-1, BRS-Rouxinol, BR 17-Gurgueia e TE97-304 G-4 também tiveram oviposição menor do que Pingo-de-ouro-1-1 e IT81 D-1045 Enramador. Somente o genótipo IT81 D-1045 Enramador foi estimulante à oviposição, enquanto Pingo-de-ouro-1-1, TVU-1593 e Patativa mostraramse neutros, e os demais genótipos foram classificados como deterrentes.

A menor preferência para oviposição pode estar relacionada a estímulos negativos produzidos pela planta. Para Costa et al. (2004), a não preferência da mosca-branca para oviposição em genótipos de feijão-caupi está possivelmente relacionada ao baixo teor de substâncias atraentes ou aos altos teores de repelentes, que influenciam o comportamento do inseto durante o processo de seleção hospedeira.

Considerando-se os ensaios de oviposição com chance e sem chance de escolha, verifica-se que TE93-244-23 F-1, TVU-36, TE97-304 G-4, BR 17-Gurgueia e BRS-Rouxinol mantiveramse sempre entre os que receberam menos ovos, o que indica os maiores níveis de resistência por não preferência para oviposição. Segundo Lara (1991), os materiais com maior nível de resistência (independentemente do tipo) devem manifestar comportamento similar em ambas as modalidades de ensaio, desde que mantidas as mesmas condições de infestação, situação corroborada pelo presente trabalho. Segundo Lourenção \& Yuki (1982), o teste sem chance de escolha tem importância prática, visto que, na maioria das vezes, extensas áreas são plantadas somente com uma cultivar, o que impede o inseto de escolher. O plantio de genótipos com menor oviposição detectada nesse teste poderia, portanto, reduzir consideravelmente a população da praga no campo.

As características físicas das superfícies foliares, como pilosidade, presença de tricomas, cerosidade, espessura, dureza e textura da epiderme são fatores que podem afetar a preferência do inseto por uma planta, tanto para alimentação quanto para oviposição (Lara, 1991). A importância dos tricomas como fonte de resistência a $B$. tabaci já foi reportada em diversos trabalhos (Lambert et al., 1995; Baldin et al., 2005; Fancelli et al., 2005). No entanto, no feijão-caupi, ambas as superfícies foliares são glabras (Simmons, 1994; Costa et al., 2004).

Em razão do potencial de danos que a mosca-branca apresenta para o cultivo de feijão-caupi e de sua expansão no mundo, os materiais avaliados no presente trabalho podem servir como base para programas de melhoramento genético, com foco no manejo da mosca-branca. Avaliações mais detalhadas quanto a aspectos químicos, físicos e morfológicos das folhas dos genótipos de feijão devem ser objeto de futuras investigações, para identificação das principais causas associadas à não preferência da mosca-branca por alguns dos genótipos avaliados. 
Tabela 5. Média \pm erro padrão do número de ovos por $\mathrm{cm}^{2}$ de Bemisia tabaci biótipo $\mathrm{B}$, índice de preferência para oviposição (IPO) e classificação dos genótipos de feijão-caupi quanto à preferência para oviposição, em ensaios com e sem chance de escolha, em casa de vegetação(1).

\begin{tabular}{|c|c|c|c|}
\hline Genótipo & Número de ovos por $\mathrm{cm}^{2}$ & IPO & Classificação \\
\hline & \multicolumn{3}{|c|}{ Com chance de escolha } \\
\hline TVU-1593 & $30,6 \pm 7,38 \mathrm{a}$ & $55,0 \pm 33,7$ & Estimulante \\
\hline Pingo-de-ouro-1-1 & $15,6 \pm 3,39 \mathrm{ab}$ & $33,1 \pm 35,9$ & Estimulante \\
\hline IT81 D 1045 Enramador & $12,5 \pm 2,47 \mathrm{abc}$ & $23,2 \pm 52,0$ & Estimulante \\
\hline TE94-309 G-9 & $10,1 \pm 7,03$ abcde & $11,3 \pm 53,1$ & Neutro \\
\hline Patativa & $10,0 \pm 2,59 \mathrm{abcde}$ & $10,7 \pm 65,8$ & Neutro \\
\hline Canapu & $8,5 \pm 2,94$ abcde & $0,0 \pm 0,0$ & Padrão \\
\hline BRS-Rouxinol & $4,1 \pm 1,01 \mathrm{cde}$ & $25,0 \pm 44,5$ & Deterrente \\
\hline BRS-Marataoã & $3,3 \pm 1,02 \mathrm{cde}$ & $34,4 \pm 33,6$ & Deterrente \\
\hline MNC99-541 F-21 & $3,3 \pm 0,77 \mathrm{cde}$ & $33,5 \pm 36,0$ & Deterrente \\
\hline BR 17-Gurgueia & $2,7 \pm 0,74 \mathrm{cde}$ & $33,7 \pm 49,7$ & Deterrente \\
\hline TE97-304 G-4 & $2,0 \pm 0,45 \mathrm{de}$ & $48,2 \pm 35,1$ & Deterrente \\
\hline BRS-Urubuquara & $1,5 \pm 0,65 \mathrm{de}$ & $64,0 \pm 38,6$ & Deterrente \\
\hline TVU-36 & $1,1 \pm 0,51 \mathrm{e}$ & $64,0 \pm 35,3$ & Deterrente \\
\hline TE93-244-23 F-1 & $1,1 \pm 0,38 \mathrm{e}$ & $66,6 \pm 29,2$ & Deterrente \\
\hline F & $10,37^{*}$ & & \\
\hline \multirow[t]{2}{*}{ CV (\%) } & 33,96 & & \\
\hline & \multicolumn{3}{|c|}{ Sem chance de escolha } \\
\hline Pingo-de-ouro-1-1 & $17,1 \pm 3,29 \mathrm{a}$ & $9,9 \pm 33,0$ & Neutro \\
\hline IT81 D-1045 Enramador & $16,3 \pm 1,26 \mathrm{a}$ & $12,8 \pm 31,8$ & Estimulante \\
\hline TVU- 1593 & $14,8 \pm 4,69 \mathrm{ab}$ & $1,8 \pm 43,4$ & Neutro \\
\hline Canapu & $14,7 \pm 4,61 \mathrm{ab}$ & $0,0 \pm 0,00$ & Padrão \\
\hline Patativa & $13,6 \pm 5,20 \mathrm{abc}$ & $4,3 \pm 40,4$ & Neutro \\
\hline BRS-Marataoã & $8,2 \pm 2,04 \mathrm{abc}$ & $25,3 \pm 34,6$ & Deterrente \\
\hline BRS-Urubuquara & $6,9 \pm 1,09 \mathrm{abc}$ & $28,5 \pm 26,0$ & Deterrente \\
\hline TE94-309 G-9 & $6,7 \pm 1,56 \mathrm{abc}$ & $33,3 \pm 14,8$ & Deterrente \\
\hline MNC99-541 F-21 & $6,5 \pm 1,27 \mathrm{abc}$ & $33,0 \pm 25,2$ & Deterrente \\
\hline TE93-244-23 F-1 & $5,1 \pm 1,08 b c$ & $42,4 \pm 27,2$ & Deterrente \\
\hline BRS-Rouxinol & $4,4 \pm 1,06 \mathrm{bc}$ & $51,2 \pm 27,0$ & Deterrente \\
\hline BR 17-Gurgueia & $4,3 \pm 0,73 \mathrm{bc}$ & $48,6 \pm 19,0$ & Deterrente \\
\hline TE97-304 G-4 & $4,2 \pm 0,70 \mathrm{bc}$ & $44,9 \pm 30,6$ & Deterrente \\
\hline TVU-36 & $3,4 \pm 0,57 \mathrm{c}$ & $56,8 \pm 17,8$ & Deterrente \\
\hline $\mathrm{F}$ & $5,46^{*}$ & & \\
\hline CV (\%) & 27,90 & & \\
\hline
\end{tabular}

${ }^{(1)}$ Médias seguidas de letras iguais, nas colunas, não diferem entre si, pelo teste de Tukey, a $5 \%$ de probabilidade. Dados originais para análise foram transformados em $(x+0,5)^{1 / 2} \cdot{ }^{(2)}$ Estimulante, valor positivo e maior que o erro padrão (EP); neutro, valor positivo ou negativo e menor que o erro padrão; deterrente, valor negativo e maior que o erro-padrão. IPO, índice de preferência para oviposição: $\mathrm{IPO}=[(\mathrm{T} P)] /[(\mathrm{T}+\mathrm{P})]$ x 100, em que: T é o número de ovos no genótipo avaliado, e P é o número de ovos no genótipo padrão.

\section{Conclusão}

OsgenótiposBRS-Urubuquara,TVU-36,TE93-244-23 F-1,BR 17-Gurgueia, MNC99-541 F-21, BRS-Marataoã, TE97-304 G-4 e BRS-Rouxinol são menos atrativos e ovopositados por Bemisia tabaci biótipo B e, portanto, são promissores para uso em programas de melhoramento genético do feijão-caupi.

\section{Agradecimentos}

À Coordenação de Aperfeiçoamento de Pessoal de Nível Superior, por concessão de bolsas; à Dra. Judith
K. Brown, da Universidade do Arizona, Tucson, AZ, EUA, pela identificação de Bemisia tabaci biótipo B; e ao Dr. Dinival Martins, Universidade Estadual Paulista, Botucatu, SP, pelo fornecimento dos dados climatológicos.

\section{Referências}

ALENCAR, J.A.A.; HAJI, F.N.P.; BLEICHER, E.; BARBOSA, F.R. Métodos gerais de controle da mosca-branca. In: HAJI, F.N.P.; BLEICHER, E. (Ed.). Avanços no manejo da mosca-branca Bemisia tabaci biótipo B (Hemiptera: Aleyrodidae). Petrolina: Embrapa Semi-Árido, 2004. p.43-49. 
BALDIN, E.L.L.; VENDRAMIM, J.D.; LOURENÇÃO, A.L. Resistência de genótipos de tomateiro à mosca-branca Bemisia tabaci (Gennadius) biótipo B (Hemiptera: Aleyrodidae). Neotropical Entomology, v.34, p.435-441, 2005.

BANZATTO, D.A.; KRONKA, S.N. Experimentação agrícola. 4.ed. Jaboticabal: Funep, 2006. 247p.

BYRNE, D.N.; BELLOWS JUNIOR, T.S. Whitefly biology. Annual Review of Entomology, v.36, p.431-457, 1991.

COSTA, N.P. da; SANTOS, T.M. dos; BOIÇA JÚNIOR, A.L. Preferência para oviposição de Bemisia tabaci biótipo-B em genótipos de caupi. Acta Scientiarum. Agronomy, v.26, p.227-230, 2004.

FANCELLI, M.; VENDRAMIM, J.D.; FRIGHETTO, R.T.S.; LOURENÇÃO, A.L. Exsudato glandular de genótipos de tomateiro e desenvolvimento de Bemisia tabaci (Genn.) (Sternorrhyncha: Aleyrodidae) biótipo B. Neotropical Entomology, v.34, p.659-665, 2005.

FAZOLIN, M.; ESTRELA, J.L.V.; LEMOS, R.N.S.; JÚNIOR, A.L.M.; FRAGOSO, D.B.; TEIXEIRA, C.A.D.; SALLET, L.A.P.; CARDOSO, S.R.S.; MEDEIROS, F.R.; TREVISAN, O.; SOUZA, F.F.; CHAGAS, E.F.; SILVA, R.Z.; LIMA, A.C.S. Insetos-praga e seus inimigos naturais. In: ZILLI, J.E.; VILARINHO, A.A.; ALVES, J.M.A. (Ed.). A cultura do feijão-caupi na Amazônia brasileira. Boa Vista: Embrapa Roraima, 2009. p.271-304.

FENEMORE, P.G. Oviposition of potato tuber moth, Phthorimaea operculella Zell (Lepidoptera: Gelechiidae) - identification of host-plant factors influencing oviposition response. New Zealand Journal of Zoology, v.7, p.435-439, 1980.

FREIRE FILHO, F.R.; RIBEIRO, V.Q.; ROCHA, SILVA, K.J.D.; NOGUEIRA, M.S.R.; RODRIGUES, E.V. Feijão-caupi no Brasil: produção, melhoramento genético, avanços e desafios. Teresina: Embrapa Meio-Norte, 2011. 84p.

JONES, D.R. Plant viruses transmitted by whiteflies. European Journal of Plant Pathology, v.109, p.195-219, 2003.

LACERDA, J.T. de; CARVALHO, R.A. Descrição e manejo integrado da mosca-branca (Bemisia spp.) transmissora de Geminivirus em culturas econômicas. Tecnologia e Ciência Agropecuária, v.2, p.15-22, 2008.

LAMBERT, A.L.; MCPHERSON, R.M.; ESPELIE, K.E. Soybean host plant resistance mechanisms that alter abundance of whiteflies (Homoptera: Aleyrodidae). Environmental Entomology, v.24, p.1381-1386, 1995.

LARA, F.M. Princípios de resistência de plantas a insetos. 2.ed. São Paulo: Ícone, 1991. 336p.

LOURENÇÃO, A.L.; YUKI, V.A. Oviposição de Bemisia tabaci (Genn.) (Homoptera: Aleyrodidae) em três variedades de soja sem chance de escolha. Bragantia, v.41, p.199-202, 1982.

MARTINEZ, S.S. O nim Azadirachta indica: natureza, usos múltiplos, produção. Londrina: IAPAR, 2011. 205p.

MELO, F.B.; CARDOSO, M.J.; SALVIANO, A.A.C. Fertilidade do solo e adubação. In: FREIRE FILHO, F.R.; LIMA, J.A. de
A.; RIBEIRO, V.Q. (Ed.). Feijão-caupi: avanços tecnológicos. Brasília: Embrapa Informação Tecnológica; Teresina: Embrapa Meio-Norte, 2005. p.231-242.

MUSA, P.D.; REN, S.-X. Development and reproduction of Bemisia tabaci (Homoptera: Aleyrodidae) on three bean species. Insect Science, v.12, p.25-30, 2005.

OLIVEIRA, C.R.R. de; FREIRE FILHO, F.R.; NOGUEIRA, M. do S. da R.; BARROS, G.B.; EIRAS, M.; RIBEIRO, V.Q.; LOPES, A.C. de A. Reação de genótipos de feijão-caupi revela resistência às coinfecções pelo Cucumber mosaic virus, Cowpea aphid-borne mosaic virus e Cowpea severe mosaic virus. Bragantia, v.71, p.59-66, 2012.

PAINTER, R.H. Insect resistance in crop plants. New York: MacMillan, 1951. 520p.

RODRIGUES, N.E.L.; BOIÇA JÚNIOR, A.L.; FARIAS, P.R. da S. Antibiose e não preferência para oviposição de Bemisia tabaci (Genn.) biótipo b (Hemiptera: Aleyrodidae) por cultivares de Vigna unguiculata (L.) WALP. Arquivos do Instituto Biológico, v.79, p.25-31, 2012.

SCHILICK-SOUZA, E.C.; BALDIN, E.L.L.; LOURENÇÃO, A.L. Variation in the host preferences and responses of Ascia monuste orseis Godart (Lepidoptera: Pieridae) to cultivars of collard greens Brassica oleracea (L.) var. acephala. Journal of Pest Science, v.84, p.429-436, 2011.

SILVA, P.H.S. da; BLEICHER, E.; CARNEIRO, J.S.; BARBOSA, F.R. Manejo da mosca-branca Bemisia tabaci biótipo B na cultura do caupi. In: HAJI, F.N.P.; BLEICHER, E. (Ed.). Avanços no manejo da mosca-branca Bemisia tabaci biótipo B (Hemiptera: Aleyrodidae). Petrolina: Embrapa Semi-Árido, 2004. p.121-129.

SILVA, P.H.S. da; CASTRO, M. de J.P. de; FREIRE FILHO, F.R. Resistência do tipo não-preferência para alimentação e oviposição de mosca-branca em genótipos de feijão-caupi. Teresina: Embrapa Meio-Norte, 2008. 4p. (Embrapa Meio-Norte. Comunicado técnico, 207).

SIMMONS, A.M. Oviposition on vegetables by Bemisia tabaci (Homoptera: Aleyrodidae): temporal and leaf surface factors. Environmental Entomology, v.23, p.381-389, 1994.

SINGH, B.B.; EHLERS, J.D.; SHARMA, B.; FREIRE FILHO, F.R. Recent progress in cowpea breeding. In: FATOKUN, C.A.; TARAWALI, S.A.; SINGH, B.B.; KORMAWA, P.M.; TAMÒ, M. (Ed.). Challenges and opportunities for enhancing sustainable cowpea production. Ibadan: International Institute of Tropical Agriculture, 2002. p.22-40.

SMITH, C.M. Plant resistance to arthropods. Dordrecht: Springer, 2005. 423p.

VENDRAMIM, J.D.; GUZZO, E.C. Resistência de plantas e a bioecologia e nutrição dos insetos. In: PANIZZI, A.R.; PARRA, J.R.P. (Ed.). Bioecologia e nutrição dos insetos: base para o manejo integrado de pragas. Brasília: Embrapa Informação Tecnológica; Londrina: Embrapa Soja, 2009. p.1055-1105.

Recebido em 5 de maio de 2012 e aprovado em 26 de setembro de 2012 